\title{
Gynophore miRNA analysis at different developmental stages in Arachis duranensis
}

\author{
Y. Shen ${ }^{1,2 *}$, Y.H. Liu ${ }^{1 *}$, X.J. Zhang ${ }^{3}$, Q. Sha ${ }^{1}$ and Z.D. Chen ${ }^{1}$ \\ ${ }^{1}$ Institute of Industrial Crops, Jiangsu Academy of Agricultural Sciences, \\ Nanjing, Jiangsu, China \\ ${ }^{2}$ Provincial Key Laboratory of Agrobiology, \\ Jiangsu Academy of Agricultural Sciences, Nanjing, Jiangsu, China \\ ${ }^{3}$ College of Agronomy and Plant Protection, Qingdao Agricultural University, \\ Qingdao, China
}

*These authors contributed equally to this study.

Corresponding author: Z.D. Chen

E-mail: chen701865@aliyun.com

Genet. Mol. Res. 15 (4): gmr15048691

Received April 4, 2016

Accepted May 17, 2016

Published October 24, 2016

DOI http://dx.doi.org/10.4238/gmr15048691

Copyright (C) 2016 The Authors. This is an open-access article distributed under the terms of the Creative Commons Attribution ShareAlike (CC BY-SA) 4.0 License.

\begin{abstract}
Peanut is one of the most important oilseed crops in the world that provides a significant amount of lipids and protein for many people. The gynophore plays an important role in gynophore development after fertilization of the peanut ovule. MicroRNAs (miRNAs) play an important role in numerous developmental and physiological plant processes. Therefore, it is essential to analyze the miRNA sequences at different gynophore stages to explore and validate gene function. Multiple small RNAs were sequenced and collected from gynophore stages A1, A2, and A3 (5, 10, and 20 days of development, respectively) for further prediction. We obtained 266 known and 357 novel miRNAs from the three different stages. Stage A3 had the largest number of reads. Genes involved in the lignin catabolic process
\end{abstract}


were identified only at stage A1. The copper ion-binding process also specifically emerged at stage A1, whereas negative regulation of biological processes occurred only in stages A2 and A3. The genes related to growth were found only at stage A3, suggesting that the gynophore may contribute to rapid development of the gynophore at this stage. Our identification and assessment of miRNAs from different gynophore stages may serve as a basis for further studies of gynophore miRNA regulation mechanisms. Some biological processes were specifically regulated at different gynophore stages indicating that miRNAs play an important role in the gynophore development.

Key words: Peanut; Gynophore; MicroRNA sequencing

\section{INTRODUCTION}

Studies have shown that gynophore has similar absorption characteristics to roots, is sensitive to light and friction, and has geotropism. A gynophore is the stalk of certain flowers that supports the gynoecium (the ovule-producing part of a flower), elevating it above the branching points of other floral parts. Gravitropism (also known as geotropism) is a turning or growth movement by a plant or fungus in response to gravity. Various hormones have shown marked differences in accumulation and distribution during different stages of gynophore development (Moctezuma, 2003). Before enlargement of the buried peanut gynophore, auxin (IAA) and abscisic acid levels rapidly increase, and ethylene is released. In addition, rapidly decreasing content of gibberellic acid (GA) results in a significantly increased expression of the enzyme gibberellin 2-oxidase, which is negatively regulated by GA (Wang et al., 2013).

MicroRNAs (miRNA) play important roles in eukaryotes by regulating target genes. After the discovery of the first miRNA (Lee et al., 1993), a large number of miRNAs have been identified and analyzed in various animals and plants that are recorded in the miRBase database (http://www.mirbase.org/, Release 16.0, September 2010) (Griffiths-Jones, 2006; Zhang et al., 2006; Griffiths-Jones et al., 2006, 2008). In recent years, transcription-based high-throughput sequencing methods have become more sophisticated to identify the function of miRNA in the peanuts (Chi et al., 2011; Deng et al., 2012). Many studies have focused on furthering our understanding of the gynophore development. Chen et al. (2013) took advantage of available transcriptome data to detect two cell-aging transcripts that significantly increased in the late stage of unburied gynophores and may participate in the abortion of gynophores on the ground. Xia et al. (2013) found that a series of transcripts, including endogenous hormones, respond to optical signals, and that morphogenesis and cell division pathway transcripts showed distinct changes before and after the burying of the gynophore. Zhu et al. (2014) compared the transcriptome of the gynophore before and after burying, and described the relevance of changes in expression and hormone levels of GA, IAA, and other hormone-related transcripts. Chen et al. (2015) detected 11 transcriptomes from different developmental stages of the gynophore, and exhaustively analyzed the transcripts associated with gravitropism and photomorphogenesis. In addition, a proteomic study including different gynophore developmental stages has also been reported (Zhu et al., 2013; Zhao et al., 2015). In summary, high-throughput sequencing has helped further our understanding of the dynamic development processes of the gynophore at the molecular and protein levels. The information

Genetics and Molecular Research 15 (4): gmr15048691 
gained from various transcription processes provides further references for gene cloning.

A shortcoming of existing studies is that high-throughput sequencing experiments have only been carried out with tetraploid-cultivated peanut (Yueyou 7 and Luhua 14). This restriction to the highly homologous tetraploid chromosomes between peanut AA and BB makes it difficult to effectively detect transcripts and to explore and validate gene function. In this study, miRNA data from different stages of gynophore development were obtained from diploid wild peanut Arachis duranensis. This information could be used to further expand the research on different developmental stages of the gynophore and effectively determine the important functional genes.

\section{MATERIAL AND METHODS}

\section{Plant materials}

Peanuts (A. duranensis) were grown in the field at Jiangsu Academy of Agricultural Sciences. Three different gynophore stages (A1: 5 days, A2: 10 days, and A3: 20 days) were collected and immediately stored in liquid nitrogen before RNA extraction. This study was approved by the Institutional Plant Care and Use Committee of Jiangsu Academy of Agricultural Sciences.

\section{RNA extraction and high-throughput sequencing}

Total RNA was obtained from the different gynophore stages (A1, A2, and A3) using Trizol agent (TaKaRa, Dalian, China), following the manufacturer instructions. Total RNA of peanut gynophores was isolated by an improved CTAB method with isopropanol instead of lithium chloride for RNA precipitation (Mi et al., 2008). Briefly, $1 \mathrm{~g}$ gynophore tissue was added to liquid nitrogen to obtain a fine powder. Subsequently, the powder was evenly mixed using $5 \mathrm{~mL}$ preheated $\left(65^{\circ} \mathrm{C}\right)$ extraction buffer $(2 \%$ CTAB, $\%$ PVP, $0.1 \mathrm{M}$ Tris-HCl, $2.0 \mathrm{M}$ $\mathrm{NaCl}, 25 \mathrm{mM}$ EDTA, 2\% beta-mercaptoethanol, $\mathrm{pH}$ 8.0). The mixture was incubated for 5 min at $65^{\circ} \mathrm{C}$ and shaken three times during the incubation time. After a short-time cooling, $2.5 \mathrm{~mL}$ isopropanol was added to the cooling mixture. After vortexing for $1 \mathrm{~min}$, the mixture was centrifuged at $13,800 \mathrm{~g}$ for $15 \mathrm{~min}$ at $4^{\circ} \mathrm{C}$. DNase was used to treat the extraction, and RNA was precipitated at $25^{\circ} \mathrm{C}$ for 10 min using $2.5 \mathrm{~mL}$ isopropanol. The extracted RNA was first resuspended with an equal volume of mixture (phenol:chloroform:isopropanol $=$ 25:24:1), followed by resuspension using an equal mixture volume (chloroform:isopropanol $=24: 1$ ). Both $0.25 \mathrm{~mL} 3 \mathrm{M} \mathrm{NaOAC}, \mathrm{pH} 5.2$, and $7.5 \mathrm{~mL}$ cooling ethanol were added to the mixture to precipitate the RNA overnight at $-20^{\circ} \mathrm{C}$. The quality of the prepared RNA from each sample was analyzed using Agilent 2100 (Agilent Technologies Co., Ltd., Palo Alto, CA, USA). The added fragmentation buffer (Ambion) was used to cut mRNA into short fragments (200-700 nt). First-strand cDNA was synthesized based on the shortfragment templates. DNA polymerase I (New England Biolabs, Beijing, China), dNTPs, RNase H (Invitrogen), and buffer were used to synthesize the second-strand cDNA. The fragments were purified using QiaQuick PCR kit and washed in EB buffer for end repair before adding polyA tails and adaptors. Fragments of suitable size were collected by agarose gel electrophoresis and amplified using polymerase chain reaction (PCR). The resulting products were sequenced by Illumina HiSeq ${ }^{\mathrm{TM}} 2000$.

Genetics and Molecular Research 15 (4): gmr15048691 


\section{Prediction of novel miRNA}

In order to predict novel gynophore miRNAs, the small RNA sequences were aligned to ESTs of peanut to collect precursor sequences. The structural features of the miRNA precursors were analyzed using the Mireap program constructed by the Beijing Genome Institute to find novel miRNA candidates. The collected structures were considered to be novel miRNA candidates when they conformed to the standard described by Allen et al. (2005). Mfold (Zuker, 2003) was used to examine the secondary structures of the collected pre-miRNA sequences. Furthermore, structures with the lowest energy were filtered for manual inspection.

\section{Target gene prediction}

The psRNATarget program (http://bioinfo3.noble.org/psRNATarget) was used to analyze the potential targets of the peanut miRNAs. The novel peanut miRNA fragments were used as custom miRNA sequences. Arachis transcript/genomic libraries (GSS, EST, and nucleotide databases) were used as custom plant databases. The analyzed genes were scored, and the standard roles used in this study were as follows: every G:U wobble pairing received 0.5 points, 2.0 points were assigned for every indel, and all non-canonical WatsonCrick pairings received 1.0 point each. The complete score of an alignment was calculated based on $20 \mathrm{nt}$. Scores of all possible consecutive $20 \mathrm{nt}$ subsequences were calculated when the fragment was longer than $20 \mathrm{nt}$, and the minimum score was collected as the total score of the query-subject alignment. Given that the targets' complementary sequence at the miRNA 5'-end is critical, mismatches other than G:U wobbles at positions 2-7 at the 5'-end received a 0.5 point deduction of the final score (Griffiths-Jones et al., 2005). The sequences whose final scores were less than 3.0 points were considered to be the miRNA targets. Redundant sequences were deleted using the Vector NTI program, when the potential target mRNA sequences were collected. The target sequences were subjected to BLASTx analysis and the functions of the potential targets were analyzed in the NCBI database.

\section{Analysis of GO pathway}

BLASTx analysis was performed using the target sequences and the NCBI database to better understand the function of the miRNA targets and the metabolic regulatory networks related to the identified peanut miRNAs. A BLASTx of the Interpro and KEGG databases was used to collect the predicted target proteins with an $E$ value of 1e-30. The target gene function and metabolic pathway of the miRNAs were verified using the best hits. Finally, we obtained molecular function, biological process, and cellular component of the target genes from the GO and Interpro database.

\section{Data analysis}

Clean reads were filtered from the raw reads obtained after sequencing, by removing sequences containing adapters. The collected reads were used for de novo assembly, which was performed using the SOAPdenovo program. Firstly, the filtered reads with suitable overlap length were combined to form contigs. Secondly, the filtered reads were re-mapped to contigs, and then scaffolds were made. Lastly, pair-end reads were used again for gap filling to

Genetics and Molecular Research 15 (4): gmr15048691 
construct unigenes. To show the alternation of each DEG during the whole development stages, their fold-change data were imported into MultiExperiment Viewer (MeV v4.8, http://www.tm4. org/mev/) for hierarchical clustering analysis with the average linkage method. The functional annotation, GO functional analysis, and KEGG pathway analysis of these unigenes were carried out using BLASTx $(E$ value $<0.00001)$ against the Nr, Swiss-Prot, KEGG, and COG databases.

\section{RESULTS}

\section{Sequence analysis}

In the present study, high-throughput sequencing was used to identify low-abundance candidate miRNAs in gynophores at different developmental stages. After filtering out lowquality tags, excluding adaptor/acceptor sequences, and removing contamination caused by adaptor-adaptor ligation, multiple-clean reads were obtained. All sequences were found to be small RNAs, including miRNA, noncoding RNA, tRNA, rRNA, snRNA, and snoRNA. Of the small RNAs, $70.62 \%$ were common to stages A1 and A2, whereas 16.28 and $13.10 \%$ were unique to stages A1 and A2, respectively. Furthermore, $60.45 \%$ of the small RNAs were common to stages $\mathrm{A} 1$ and $\mathrm{A} 3$, whereas 15.72 and $23.83 \%$ were unique to stages $\mathrm{A} 1$ and $\mathrm{A} 3$, respectively. Finally, $64.17 \%$ of the small RNAs were common to stages A2 and A3, $12.33 \%$ were unique to stage $\mathrm{A} 2$ and $23.50 \%$ were unique to stage $\mathrm{A} 3$ (Table 1). The numbers and kinds of small RNAs identified in the gynophore at different stages are shown in Table 1. A large number of known miRNAs were detected in all three developmental stages of the peanut gynophore. More miRNAs were found in stage A2 than in stages A1 or A3. Interestingly, stage A1 contained the most different kinds of miRNAs, whereas stage A3 had the fewest kinds of miRNAs. The length distribution of small RNAs at different stages often determines their roles in the particular stage and biogenetic machines. As shown in Figure 1, the majority of the small RNAs in the stage A1, A2, and A3 libraries were $21 \mathrm{nt}$ long and accounted for 29.9, 34.0 , and $32.5 \%$ of the total sequence numbers, respectively. In addition, the number of small RNAs (24 nt) from stages A1, A2, and A3 were 26.4, 22.7, and 30.4\%, respectively, of the total number of RNAs. The 21 and 24 nt small RNAs were the most prevalent in the gynophore, which was consistent with the results reported for other peanut tissues (Chi et al., 2011). There were more $21 \mathrm{nt}$ small RNAs than 24 nt RNAs in gynophore. Furthermore, as found in other studies (Chi et al., 2011), the high percentage of $24 \mathrm{nt}$ small RNAs in the peanut gynophore could be indicative of the complexity of the peanut genome.

Table 1. Total and unique gynophore stages (A1, A2, and A3).

\begin{tabular}{l|c|c|c|c|c|c}
\hline \multirow{2}{*}{ Category } & \multicolumn{3}{|c|}{ Total } & \multicolumn{2}{c}{ Unique } \\
\cline { 2 - 7 } & $\mathrm{A} 1$ & $\mathrm{~A} 2$ & $\mathrm{~A} 3$ & $\mathrm{~A} 1$ & $\mathrm{~A} 2$ & $\mathrm{~A} 3$ \\
\hline Intro sense & 524566 & 397067 & 529940 & 265675 & 205660 & 280197 \\
\hline Intro antisense & 257063 & 182697 & 295128 & 138474 & 104909 & 148491 \\
\hline Exon sense & 303767 & 303429 & 367802 & 117457 & 113322 & 118786 \\
\hline Exon antisense & 162741 & 163788 & 263940 & 73265 & 61219 & 89830 \\
\hline snoRNA & 4447 & 6665 & 5956 & 1510 & 1770 & 1836 \\
\hline snRNA & 7294 & 12175 & 10548 & 1755 & 2145 & 2381 \\
\hline Known miRNA & 1265723 & 1284734 & 1175955 & 3275 & 3159 & 2838 \\
\hline miRNA & 209469 & 269289 & 212947 & 16439 & 15729 & 15147 \\
\hline tRNA & 635578 & 480252 & 624491 & 38593 & 53824 & 57599 \\
\hline rRNA & 2275873 & 3132040 & 1874242 & 223665 & 268616 & 230163 \\
\hline rRNAetc & 92534 & 105705 & 84033 & 15719 & 14795 & 15829 \\
\hline unann & 8205594 & 7634949 & 8972250 & 3986769 & 3193330 & 4449319 \\
\hline
\end{tabular}

Genetics and Molecular Research 15 (4): gmr15048691 

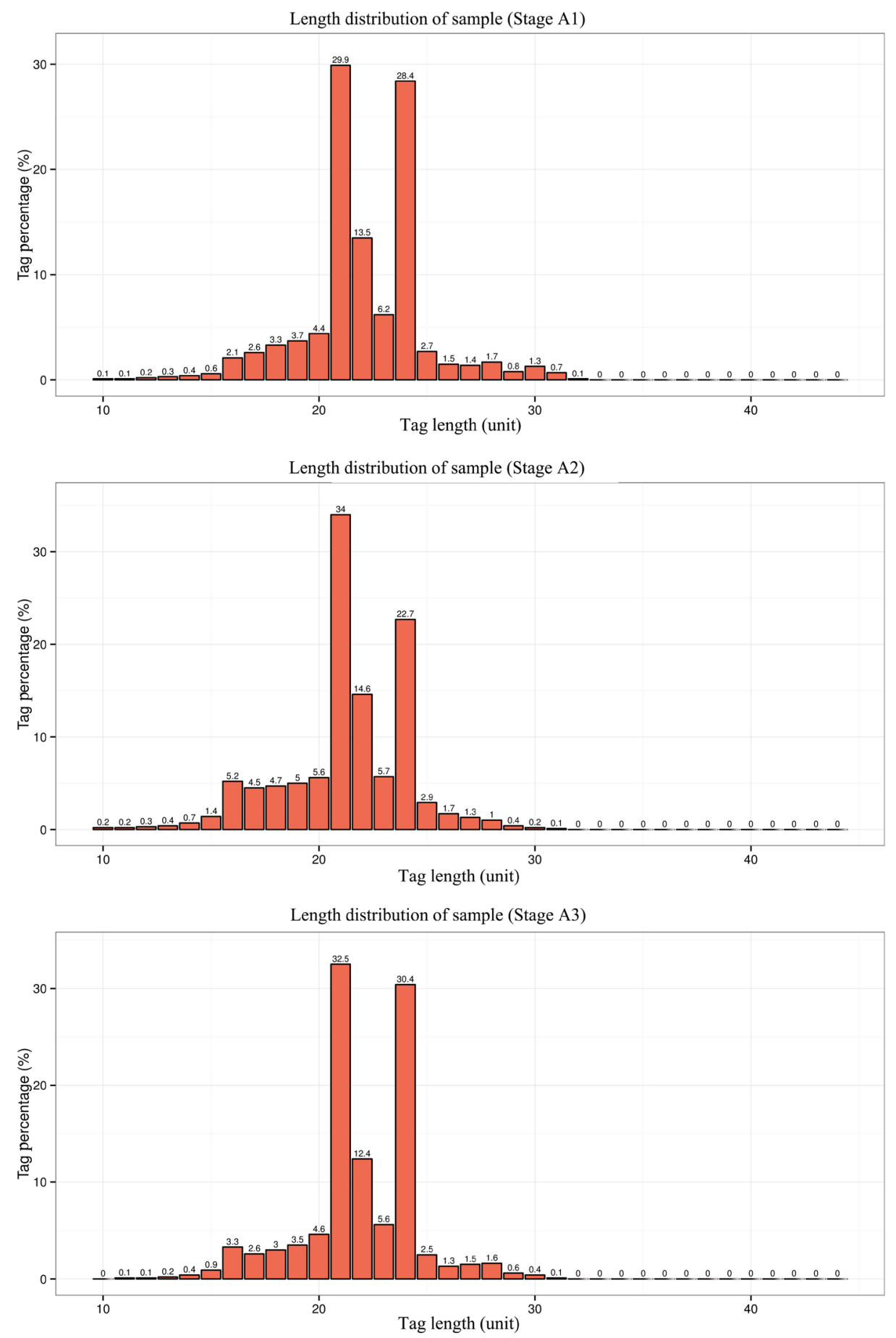

Figure 1. Tag length distribution of the three different gynophore stages.

Genetics and Molecular Research 15 (4): gmr15048691 


\section{Known and novel miRNAs}

There were 266 known miRNAs detected in the three different stages of the gynophore (Table S1). As shown in previous studies, most miRNAs from the gynophore were highly conserved in different plant species (Sunkar et al., 2008), which indicates that the ancient regulatory pathways of conserved miRNAs are present in peanut too. miRNA members from different stages of the gynophore were compared with each other and great differences were found among them. The miRNAs miR166d-5p, miR7785-3p, miR5713, miR1080, miR8578.1, and miR482c-5p were present in more than 1000 reads in stage A1 but were not found at all in stages A2 and A3. Likewise, three miRNAs (miR166a, miR482e, and miR9722) were identified in more than 1000 reads from stage A2, whereas they were never observed in stages A1 and A3. The known miRNA, miR166u, was sequenced in more than 300,000 reads in stage A3, which indicates that this miRNA might play a significant and essential role in forming the peanut gynophore. Furthermore, a cluster analysis was performed to visualize the read differences between the three stages of the gynophore. As shown in Figure 2A, many known miRNAs showed great differences between reads of any two stages, suggesting that some play specific roles in different gynophore stages. A cluster analysis was used to analyze the differences of reads among the three gynophore stages (Figure 2B).
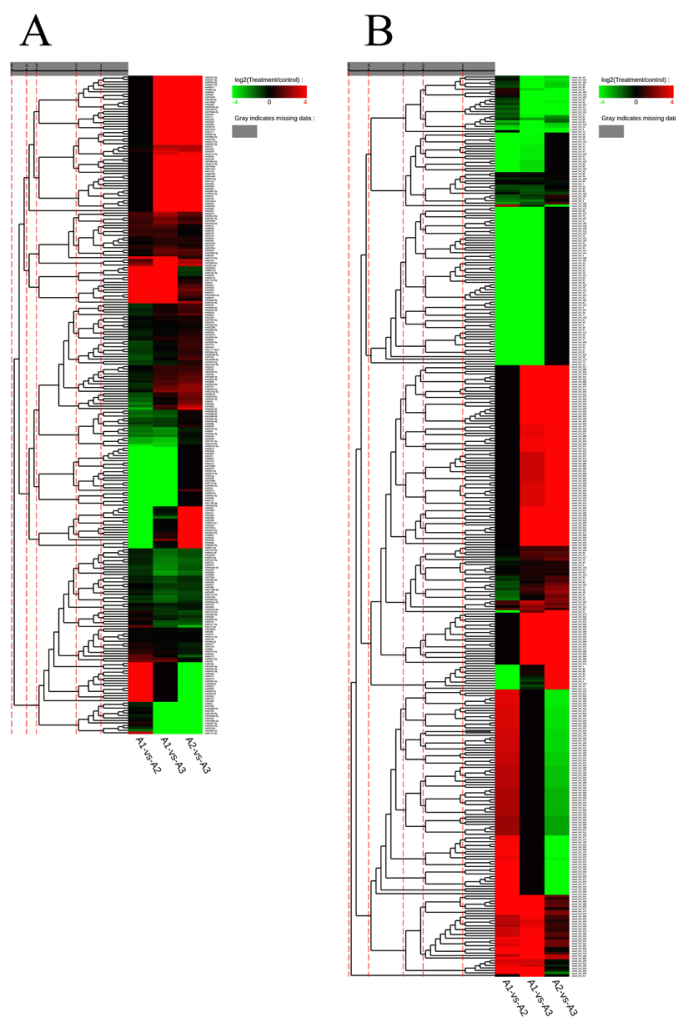

Figure 2. Cluster analysis of miRNAs from three different gynophore stages (A1, A2, and A3) of known (A) and novel (B) miRNAs. Phylogeny scale bar shows the different levels of clusterings of miRNAs by their expression patterns in three stages.

Genetics and Molecular Research 15 (4): gmr15048691 
Many novel miRNAs showed great read differences between any two stages, which suggests that there were some specific roles of the miRNAs in the different gynophore stages. In order to compare the miRNA expression from the three gynophore stages, the number of reads mapped to a gene was analyzed. As shown in Figure 3, the stage A1 score was the highest and stage A3 was the lowest. The number of reads mapped to a gene from stage A1 was higher than the other two stages suggesting that the miRNA function in stage A1 may be more complex compared to the other two stages. These results indicate that different miRNAs had different expression patterns, which may be due to stage-specific expression.
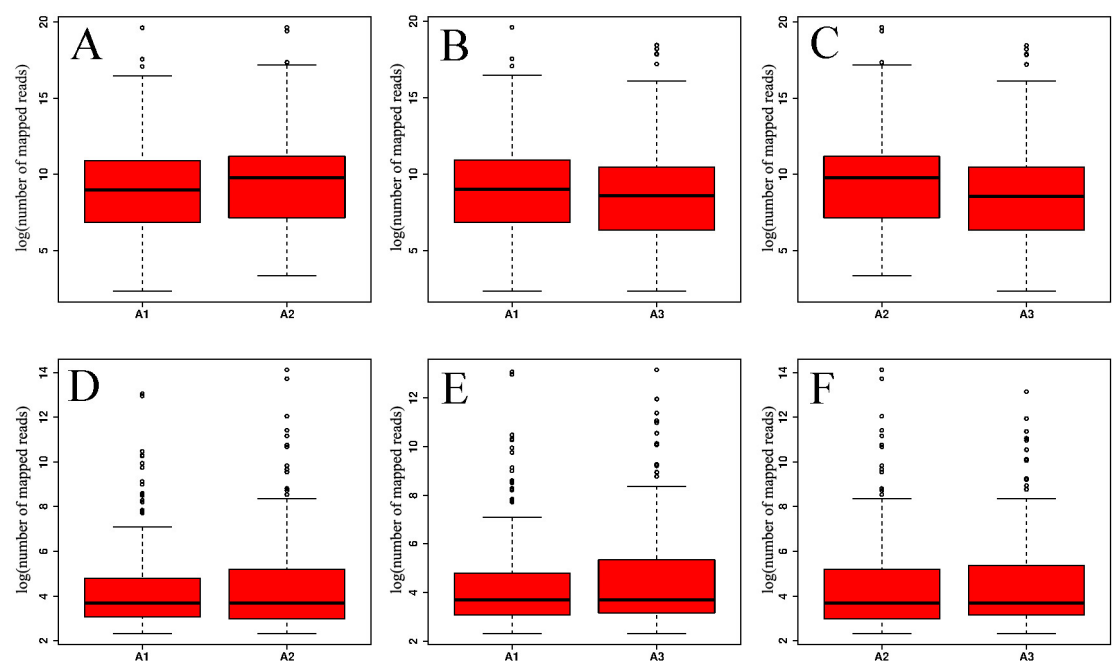

Figure 3. Number of reads to one gene from different gynophore stages (A1, A2, and A3). The contrast between stages A1 and A2 of known (A) and novel (D) miRNAs, between stages A1 and A3 (B: known, E: novel miRNAs), and between stages $\mathrm{A} 2$ and $\mathrm{A} 3$ (C: known, F: novel miRNAs).

The identified novel miRNAs of stages A1, A2, and A3 are listed in Table S1. A total of 357 novel miRNAs were found according to criteria constructed by Allen et al. (2005). Most of the novel miRNAs were sequenced with less than 100 reads, which indicates that most predicted novel miRNAs exhibited low expression levels. Moreover, the appearances and reads of the novel miRNAs in stages A1, A2, and A3 exhibited imbalance. There were 155 novel miRNAs predicted successfully from stage A1, whereas 174 and 181 novel miRNAs were found in stages A2 and A3, respectively. The result of the prediction showed that novel mir_14, novel_mir_76, and novel_mir_113 were sequenced with more than 200 reads and only detected in gynophore stage A1. The novel mir 240 and novel mir 265 were sequenced with more than 100 reads and only detected in stage A2, whereas novel_mir_313, novel_ mir_310, novel_mir_289, and novel_mir_306 were sequenced with more than 100 reads and only detected in stage A3. Furthermore, novel_mir_73 was sequenced with more than 300 reads and found in stages A1 and A2, novel_mir_131 was sequenced with more than 200 reads and found in stages A1 and A3, and novel_mir_270, novel_mir_237, novel_mir_215, novel_ mir_261, and novel_mir_227 were sequenced with more than 100 reads and found in stages A2 and A3. The number of reads mapped to a gene was analyzed to compare the miRNA expression from the three gynophore stages. For novel miRNAs, no obvious differences 
were examined in the numbers of reads mapped to a gene among the three samples (Figure $3 \mathrm{D}, \mathrm{E}$ and $\mathrm{F}$ ). The differences in novel miRNAs from different stages may suggest that they play different roles under different growth conditions, in specific tissues, or during different gynophore developmental stages.

\section{Prediction of function}

In order to fully investigate the biological functions of the known and novel miRNAs, GO analysis was used to investigate the gene ontology of all targets regulated by the miRNAs (Du et al., 2010). The terms from different stages are listed in Table S2. The terms were divided into three categories; biological process, cellular component, and molecular function. For known miRNAs, we found that many genes were involved in the lignin catabolic process at stage A1, while no such genes were detected in stages A2 and A3. This suggests that the lignin catabolic process is important to insert the gynophore into the ground, since lignin hardens the gynophore enough to pierce the soil surface. The cellular component demonstrated that 46 genes were involved in apoplast formation at stage A1 but not in stages A2 and A3. This indicates that the apoplast development process is necessary for stage A1 to develop into stage A2. A large number of genes were involved in different binding processes in all three developmental stages. Many genes participated in the copper ion-binding process of stage A1, while none of these genes was found in stages A2 and A3. This indicates that the copper ion-binding process might play an important role in preparation before developing the gynophore in stage A3. The genes involved with protein kinase activity were fully functioning in stage A2, suggesting that protein kinases at this stage were more active than at the other two stages. As listed in Table S2, novel miRNA terms from stages A1, A2, and A3 were collected to analyze the biological functions. Several terms relating to energy consumption (ATP metabolic process, proton transport, and ATP hydrolysis-coupled proton transport) were found only at stage A3, which suggests that stage A3 may be a rapid development phase of the gynophore. In addition, genes that negatively regulate biological process were specific to stages A2 and A3, whereas no such genes were filtered in stage A1 (Figure 4).

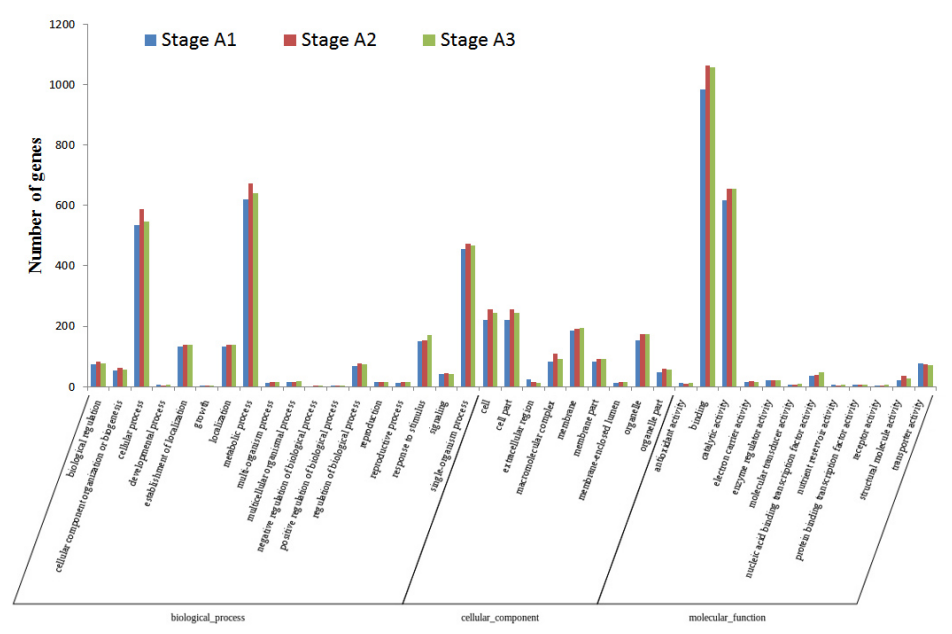

Figure 4. Function of known miRNAs from different gynophore stages (A1, A2, and A3).

Genetics and Molecular Research 15 (4): gmr15048691 
This suggests that some biological processes of the reproductive process at stage A1 may be suppressed in stages A2 and A3. There were fewer genes related to nutrient reservoir activity in stage $\mathrm{A} 2$ than in stages $\mathrm{A} 1$ and $\mathrm{A} 3$, indicating that stage $\mathrm{A} 2$ might be the consuming phase of the gynophore. As shown in Figure 5, the biological functions of novel miRNAs were similar to known miRNAs. The genes involved in growth processes were found only in stage A3, indicating that the rapid development of the gynophore occurs during stage A3.

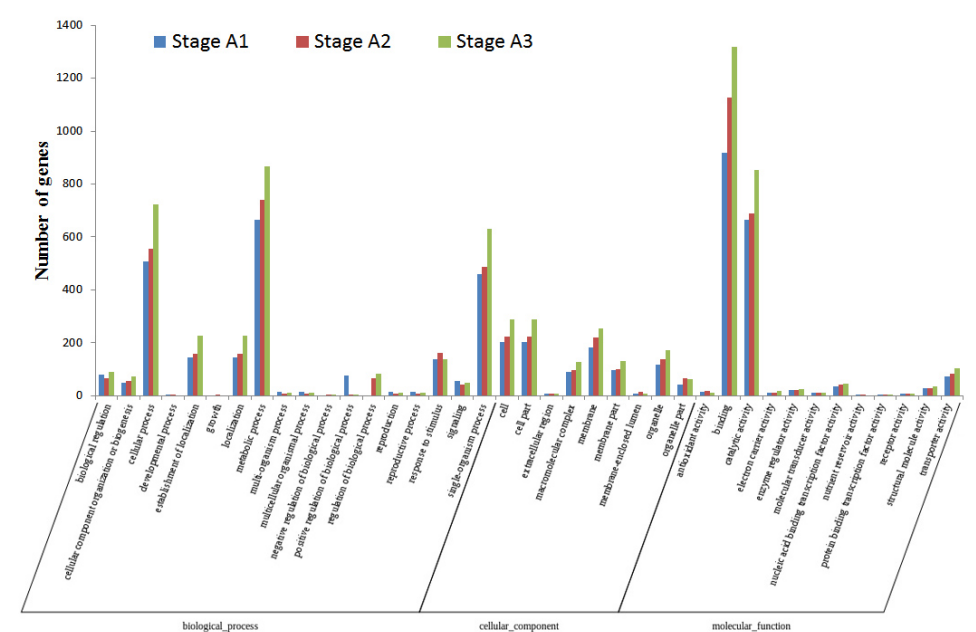

Figure 5. Function of novel miRNAs from different gynophore stages (A1, A2, and A3).

Following the GO analysis, a KEGG analysis was used to construct a pathway enrichment of the predicted known and novel miRNA target genes (Table S3 $)$. Some metabolism networks of known miRNAs were analyzed, including cytosolic DNA-sensing pathway, RNA polymerase, Epstein-Barr virus infection, plant-pathogen interaction, and pyrimidine metabolism. The metabolism networks of novel miRNAs were found, including pathogenic Escherichia coli infection, nucleotide excision repair, mismatch repair, homologous recombination, and lipid metabolism.

\section{DISCUSSION}

Studies of miRNAs in tetraploid peanut have been reported in the past several years (Chi et al., 2011). However, no study has been carried out on diploid wild peanut, which could provide a deeper understanding of transcripts and gene functions. To date, many miRNAs in the peanut gynophore have been identified and studied using computational and direct cloning approaches (Sunkar and Jagadeeswaran, 2008; Zhao et al., 2010), but the identity and functions of most miRNAs in the gynophore are still largely unknown. After removing low-quality tags, excluding adaptor/acceptor sequences, and removing contamination formed by the adaptor-adaptor ligation, many small RNAs were obtained in the present study. In accordance with previous studies (Griffiths-Jones, 2006; Griffiths-Jones et al., 2006; Chi et al., 2011), the filtered small RNAs included miRNA, noncoding RNA, tRNA, rRNA, snRNA, and snoRNA. There were fewer miRNAs in the gynophore than in the whole plant (Zhao et al., 2010), suggesting that the gynophore only had some of the functions found in the entire

Genetics and Molecular Research 15 (4): gmr15048691 
peanut plant. We found that the length distributions of small RNAs in different stages of the gynophore were similar to each other. However, differences in small RNA composition often reflect specific roles in particular tissues or species. The $21 \mathrm{nt}$ small RNAs detected in this study were different compared with those observed in the whole peanut plant (Chi et al., 2011), which indicates that the small RNAs might play a specific role in the gynophore tissue. In addition, the high percentage of $24 \mathrm{nt}$ small RNAs could reflect the complexity of the peanut gynophore genome, because $24 \mathrm{nt}$ siRNAs are known to be susceptible to heterochromatin modification, especially for a genome with a high number of repetitive sequences (Herr, 2005; Vazquez, 2006).

Evidence for the existence of 266 known miRNAs and 357 novel miRNAs in the three different gynophore development stages was identified using high-throughput Solexa technology. As shown in previous studies (Sunkar and Jagadeeswaran, 2008), most miRNAs from the gynophore are highly conserved in different plant species, indicating that ancient regulatory pathways of conserved miRNAs are present in the peanut gynophore ( $\mathrm{Li}$ et al., 2016). miRNA members of different gynophore stages were compared with each other and great differences were found between any two stages. This suggests that the miRNAs may play different roles that are specific to the different stages. The results of the miRNA analysis indicated that different members also showed differences in expression. This was probably due to expression that was specific to different developmental stages. As with the known miRNAs, many novel miRNAs showed great differences in reads between any of two stages, which also support the notion that some miRNAs play roles that are specific to the different stages of the gynophore development. The number of reads mapped to a gene was highest at stage A3 suggesting that the functions of novel miRNAs at stage A3 may be more complex compared to those in the other two stages. The differences of novel miRNAs at the different stages may suggest specific roles related to different growth conditions, specific tissues, or different developmental stages of the gynophore.

A more accurate process of target identification is important to find and define potential functions for miRNAs in plants. The results found here indicated that some of the analyzed targets of conserved miRNAs of peanut had a conserved function with miRNA targets in Arabidopsis thaliana (Griffiths-Jones et al., 2006). The miRNA target sequences were highly conserved among a variety of wild plants, as shown by Floyd and Bowman (2004). As described in earlier reports, many of the targets in the peanut gynophore are plant-specific transcription factors. However, some targets of the novel miRNAs were found to differ from A. thaliana and Oryza sativa genes, which suggests that these targets could be regarded as peanut-specific processes (Yao et al., 2007). It will be valuable to assess the functions of these predicted target genes in the peanut gynophore.

In the present study, we used GO analysis to predict the functions of miRNAs collected by high-throughput sequencing technology. The genes involved in the lignin catabolic process were found in stage A1 but not in stages A2 and A3, which suggests that this is a stagespecific process. This process is important because lignin makes the gynophore hard enough to pierce the soil surface. In addition, copper ion binding was specifically found in stage A1, providing the precondition for the subsequent $\mathrm{A} 2$ and $\mathrm{A} 3$ gynophore stages because ions play an important role in resisting ground pathogens. At stage A3, several processes involved in energy consumption were detected, whereas no such processes were found in stages A1 or A2. This difference suggests that the biological metabolism of stage A3 is vigorous because of the increased growth rate of the gynophore. Interestingly, the genes that negatively regulate biological processes were found only in stages A2 and A3, suggesting that some biological

Genetics and Molecular Research 15 (4): gmr15048691 
processes may be suppressed during these stages. The genes involved in growth were found only in stage A3, indicating that the gynophore may grow rapidly in stage A3 to develop into a gynophore. Moreover, the genes of fatty acids, lipoxygenase, and FAD-binding domaincontaining protein were found only in stage $\mathrm{A} 3$, which indicates that fatty acid accumulation and seed development are initiated at this stage (Zhao et al., 2010; Zhu et al., 2013).

Cultivated peanuts that are rich in lipid and protein are globally important oilseed crops (Chen et al., 2010). In our study, we investigated miRNAs that may play an important role in regulating biological processes involved in the lipid biosynthesis. Our results suggested that the genes involved in the lignin catabolic process may be specifically regulated in stage A1 of gynophore development. The copper ion-binding process was predicted to occur at stage A1 but not at stages A2 and A3. Many genes involved in apoplast development were found only in stage A1. Ether lipid metabolism was regulated by novel miRNAs at stage A3. These results demonstrate that miRNAs at different stages may play specific roles during the gynophore development of the peanut. In addition, our quantitative analyses of key miRNAs are important for furthering our understanding of differences in the three developmental stages. Quantitative analyses and related clone detection will be the focus of further studies.

To conclude, we identified large numbers of miRNAs from three different stages of gynophore development, analyzed their expressions, and predicted the potential targets. It will be important for future studies to predict the collected miRNAs and analyze their targets, because these could provide a better understanding of the functional relationship and mechanisms of miRNAs in the regulation network.

\section{Conflicts of interest}

The authors declare no conflict of interest.

\section{ACKNOWLEDGMENTS}

Research supported by the Self-Directed Innovation Fund of Agricultural Science and Technology in Jiangsu Province, China (grant \#CX(13)5006). We thank Gene Pioneer Biotechnologies for their help with the bioinformatic analyses.

\section{REFERENCES}

Allen E, Xie Z, Gustafson AM and Carrington JC (2005). microRNA-directed phasing during trans-acting siRNA biogenesis in plants. Cell 121: 207-221.http://dx.doi.org/10.1016/j.cell.2005.04.004

Chen X, Zhu W, Azam S, Li H, et al. (2013). Deep sequencing analysis of the transcriptomes of peanut aerial and subterranean young pods identifies candidate genes related to early embryo abortion. Plant Biotechnol. J. 11: 115127.http://dx.doi.org/10.1111/pbi.12018

Chen X, Yang Q, Li H, Li H, et al. (2015). Transcriptome-wide sequencing provides insights into geocarpy in peanut (Arachis hypogaea L.). Plant Biotechnol. J. 14: 1215-1224. http://dx.doi.org/10.1111/pbi.12487

Chen ZB, Wang ML, Barkley NA and Pittman RN (2010). A simple allele-specific PCR assay for detecting FAD2 alleles in both A and B genomes of the cultivated peanut for high-oleate trait selection. Plant Mol. Biol. Rep. 28: 542-548. http://dx.doi.org/10.1007/s11105-010-0181-5

Chi X, Yang Q, Chen X, Wang J, et al. (2011). Identification and characterization of microRNAs from peanut (Arachis hypogaea L.) by high-throughput sequencing. PLoS ONE 6: e27530.http://dx.doi.org/10.1371/journal.pone.0027530

Deng X, Li Z and Zhang W (2012). Transcriptome sequencing of Salmonella enterica serovar Enteritidis under desiccation and starvation stress in peanut oil. Food Microbiol. 30: 311-315.http://dx.doi.org/10.1016/j.fm.2011.11.001

Genetics and Molecular Research 15 (4): gmr15048691 
Du Z, Zhou X, Ling Y, Zhang Z, et al. (2010). agriGO: a GO analysis toolkit for the agricultural community. Nucleic Acids Res. 38: W64-70. http://dx.doi.org/10.1093/nar/gkq310

Floyd SK and Bowman JL (2004). Gene regulation: ancient microRNA target sequences in plants. Nature 428: 485-486. http://dx.doi.org/10.1038/428485a

Griffiths-Jones S (2006). miRBase: the microRNA sequence database. Methods Mol. Biol. 342: 129-138.

Griffiths-Jones S, Moxon S, Marshall M, Khanna A, et al. (2005). Rfam: annotating non-coding RNAs in complete genomes. Nucleic Acids Res. 33: D121-124. http://dx.doi.org/10.1093/nar/gki081

Griffiths-Jones S, Grocock RJ, van Dongen S, Bateman A, et al. (2006). miRBase: microRNA sequences, targets and gene nomenclature. Nucleic Acids Res. 34: D140-144. http://dx.doi.org/10.1093/nar/gkj112

Griffiths-Jones S, Saini HK, van Dongen S and Enright AJ (2008). miRBase: tools for microRNA genomics. Nucleic Acids Res. 36: D154-158. http://dx.doi.org/10.1093/nar/gkm952

Herr AJ (2005). Pathways through the small RNA world of plants. FEBS Lett. 579: 5879-5888. http://dx.doi.org/10.1016/j. febslet.2005.08.040

Lee RC, Feinbaum RL and Ambros V (1993). The C. elegans heterochronic gene lin-4 encodes small RNAs with antisense complementarity to lin-14. Cell 75: 843-854.http://dx.doi.org/10.1016/0092-8674(93)90529-Y

Li M, Zhao SZ, Zhao CZ, Zhang Y, et al. (2016). Cloning and characterization of SPL-family genes in the peanut (Arachis hypogaea L.). Genet. Mol. Res. 15: gmr.15017344.

Mi S, Cai T, Hu Y, Chen Y, et al. (2008). Sorting of small RNAs into Arabidopsis argonaute complexes is directed by the 5' terminal nucleotide. Cell 133: 116-127.http://dx.doi.org/10.1016/j.cell.2008.02.034

Moctezuma E (2003). The peanut gynophore: a developmental and physiological perspective. Can. J. Bot. 81: 183-190. http://dx.doi.org/10.1139/b03-024

Sunkar R and Jagadeeswaran G (2008). In silico identification of conserved microRNAs in large number of diverse plant species. BMC Plant Biol. 8: 37. http://dx.doi.org/10.1186/1471-2229-8-37

Sunkar R, Zhou X, Zheng Y, Zhang W, et al. (2008). Identification of novel and candidate miRNAs in rice by high throughput sequencing. BMC Plant Biol. 8: 25.http://dx.doi.org/10.1186/1471-2229-8-25

Vazquez F (2006). Arabidopsis endogenous small RNAs: highways and byways. Trends Plant Sci. 11: 460-468. http:// dx.doi.org/10.1016/j.tplants.2006.07.006

Wang C, Li C, Hou L, Liu X, et al. (2013). Cloning and expression analysis of Gibberellin 2-Oxidase gene from peanut (Arachis hypogaea L). Shandong Agric. Sci. 45: 14-18.

Xia H, Zhao C, Hou L, Li A, et al. (2013). Transcriptome profiling of peanut gynophores revealed global reprogramming of gene expression during early pod development in darkness. BMC Genomics $14: 517$.http://dx.doi.org/10.1186/1471$\underline{2164-14-517}$

Yao Y, Guo G, Ni Z, Sunkar R, et al. (2007). Cloning and characterization of microRNAs from wheat (Triticum aestivum L.). Genome Biol. 8: R96. http://dx.doi.org/10.1186/gb-2007-8-6-r96

Zhang B, Pan X, Cannon CH, Cobb GP, et al. (2006). Conservation and divergence of plant microRNA genes. Plant J. 46: 243-259. http://dx.doi.org/10.1111/j.1365-313X.2006.02697.x

Zhao CZ, Xia H, Frazier TP, Yao YY, et al. (2010). Deep sequencing identifies novel and conserved microRNAs in peanuts (Arachis hypogaea L.). BMC Plant Biol. 10: 3. http://dx.doi.org/10.1186/1471-2229-10-3

Zhao CZ, Zhao SZ, Hou L, Xia H, et al. (2015). Proteomics analysis reveals differentially activated pathways that operate in peanut gynophores at different developmental stages. BMC Plant Biol. 15: 188. http://dx.doi.org/10.1186/s12870$\underline{015-0582-6}$

Zhu W, Zhang E, Li H, Chen X, et al. (2013). Comparative proteomics analysis of developing peanut aerial and subterranean pods identifies pod swelling related proteins. J. Proteomics 91: 172-187. http://dx.doi.org/10.1016/j. jprot.2013.07.002

Zhu W, Chen X, Li H, Zhu F, et al. (2014). Comparative transcriptome analysis of aerial and subterranean pods development provides insights into seed abortion in peanut. Plant Mol. Biol. 85: 395-409. http://dx.doi.org/10.1007/s11103-014-0193-x

Zuker M (2003). Mfold web server for nucleic acid folding and hybridization prediction. Nucleic Acids Res. 31: $3406-$ 3415. http://dx.doi.org/10.1093/nar/gkg595

\section{Supplementary material}

Table S1. Reads of known and novel miRNAs from the three different gynophore stages.

Table S2. GO analysis of known and novel miRNAs from three different gynophore stages.

Table S3. Predictions of known and novel miRNAs' target genes by KEGG analysis).

Genetics and Molecular Research 15 (4): gmr15048691 